PROCEEDINGS OF THE

AMERICAN MATHEMATICAL SOCIETY

Volume 125, Number 9, September 1997, Pages 2753-2757

S $0002-9939(97) 03924-5$

\title{
ADJOINT ACTION OF A FINITE LOOP SPACE
}

\author{
NORIO IWASE \\ (Communicated by Thomas Goodwillie) \\ Dedicated to the memory of Kiyono Iwase
}

\begin{abstract}
Adjoint actions of compact simply connected Lie groups are studied by A. Kono and K. Kozima based on the series of studies on the classification of compact Lie groups and their cohomologies. At odd primes, there is a simpler homotopy theoretic approach that will prove the results of Kono and Kozima for any finite loop spaces. However, there are some technical difficulties at the prime 2 .
\end{abstract}

\section{INTRODUCTION}

For a connected topological group $G$, the loop group $\Lambda G=\left\{u: S^{1} \rightarrow G\right\}$ is homeomorphic to the product group $G \times \Omega G$, where $\Omega G$ denotes the subspace of loops that start and end at the unit $e \in G$. However, the multiplication of $\Lambda G$ is different from that of $G \times \Omega G$, unless $G$ is abelian. The difference can be described by the adjoint action of $G$ on $\Omega G$, say $A d: G \times \Omega G \rightarrow \Omega G$ by $A d(g, \ell)(t)=g \ell(t) g^{-1}$. Kono and Kozima [5] studied the difference in terms of the cohomology of the classifying space for $G$ a compact simple Lie group. In this paper, our approach is rather homotopy theoretic and even simple.

\section{MAIN THEOREMS}

Let $G$ be a connected topological group with the homotopy type of a finite $\mathrm{CW}$ complex and let $p$ be a prime. We have the following result, which is due to Hubbuck's Torus Theorem.

Theorem 2.1. If the inclusion $G \rightarrow B \Lambda G$ has a homotopy left inverse: $B \Lambda G \rightarrow$ $G$, then $G$ has the homotopy type of a torus of some dimension $\geq 0$ and $B \Lambda G$ is homotopy equivalent to the product $B G \times G$.

The following results are obtained by assuming homological properties.

Theorem 2.2. For an odd prime $p$, the following four conditions are equivalent if $G$ is 1-connected.

i) The induced homomorphism $j^{*}: H^{*}\left(B \Lambda G ; \mathbb{F}_{p}\right) \rightarrow H^{*}\left(G ; \mathbb{F}_{p}\right)$ is surjective.

Received by the editors November 21, 1995 and, in revised form, March 21, 1996.

1991 Mathematics Subject Classification. Primary 55P45, 55Q25; Secondary 55N20.

Key words and phrases. Loop group, loop space.

This research was supported by Grant-in-Aid for Scientists (C)08640125 from the Ministry of Education, Science and Culture of Japan.

(C)1997 American Mathematical Society 
ii) The Pontryagin ring $H_{*}\left(G ; \mathbb{F}_{p}\right)$ is a commutative Hopf algebra. In other words, the selfadjoint action induces the trivial action

$$
a d_{*}=p r_{2_{*}}: H_{*}\left(G ; \mathbb{F}_{p}\right) \otimes H_{*}\left(G ; \mathbb{F}_{p}\right) \rightarrow H_{*}\left(G ; \mathbb{F}_{p}\right) .
$$

iii) The Hopf algebra $H^{*}\left(G ; \mathbb{F}_{p}\right)$ is primitively generated.

iv) There is an $H^{*}\left(B G ; \mathbb{F}_{p}\right)$-module isomorphism

$$
H^{*}\left(B \Lambda G ; \mathbb{F}_{p}\right) \cong H^{*}\left(B G \times G ; \mathbb{F}_{p}\right) .
$$

Theorem 2.3. Under the same hypothesis as in Theorem 2.2, the conditions i), ii), iii) and iv) are also equivalent to any of the following three conditions:

v) The integral homology $H_{*}(G ; \mathbb{Z})$ has no p-torsion.

vi) The adjoint action induces the trivial action

$$
A d_{*}=p r_{2 *}: H_{*}\left(G ; \mathbb{F}_{p}\right) \otimes H_{*}\left(\Omega G ; \mathbb{F}_{p}\right) \rightarrow H_{*}\left(\Omega G ; \mathbb{F}_{p}\right) .
$$

vii) There is an isomorphism of algebras $H^{*}\left(B \Lambda G ; \mathbb{F}_{p}\right) \cong H^{*}\left(B G \times G ; \mathbb{F}_{p}\right)$.

In [5], Kono and Kozima proved the above theorems for compact Lie groups. For odd primes, we only assume that $G$ is a finite topological group or loop space.

The proofs of the above two theorems suggest that Theorem 2.2 is true even if $p=2$.

The only difficulty lies in proving that i)-iii) imply iv). And if it holds, this would visualize the 'primitivity'. At the prime 2 , the conditions given in Theorem 2.2 are clearly weaker than those in Theorem 2.3, since there exist compact Lie groups whose homology has 2 -torsion and whose cohomology mod 2 is primitively generated.

\section{KEY LEMMA}

Let us recall that the classifying space of $G \times \Omega G$ has the homotopy type of $B G \times G$. Also the classifying space of $\Lambda G$ is given by $B \Lambda G=E G \times{ }_{G} G$, where $E G$ denotes the total space of the universal principal $G$-bundle, and the (left) action of $G$ on $E G$ is the diagonal action and that on $G$ is the selfadjoint (left) action ad: $G \times G \rightarrow G$ by $\operatorname{ad}(g, h)=g h g^{-1}$. Thus there is the following fibering:

$$
G \stackrel{j}{\rightarrow} B \Lambda G=E G \times{ }_{G} G \stackrel{p}{\rightarrow} B G,
$$

where the projection $p$ has a cross-section $s: B G \rightarrow E G \times{ }_{G} G$, since the adjoint action leaves the unit fixed.

Now the proofs of the main theorems are straightforward if one notices the following lemma.

Lemma 3.1. Let $\mu: G \times G \rightarrow G$ be the multiplication of the group $G$, and $T: G \times G$ $\rightarrow G \times G$ the switching mapping. Then we have the homotopy relation $j \circ \mu \circ T \sim j \circ \mu$, where $j$ denotes the inclusion $G \rightarrow B \Lambda G$.

Proof. The total space $E G$ of the universal principal $G$-bundle is defined to be the infinite union of the $n$-fold join $E^{n} G=G * G * \cdots * G$ of $G$. Hence we have the subspaces $E^{2} G=I \times G \times G / \sim \subseteq E G$. Thus it is sufficient to show the homotopy relation $j_{0} \circ \mu \circ T \sim j_{0} \circ \mu$, where $j_{0}$ denotes the inclusion $G \rightarrow E^{2} G \times{ }_{G} G$. 
Now, let us define a homotopy $H: I \times G \times G \rightarrow E^{2} G \times{ }_{G} G$ by

$$
\begin{aligned}
& H(t, g, h)=[(1-2 t) \cdot e+2 t \cdot e ; h g], \quad t \leq \frac{1}{2}, \\
& H(t, g, h)=[(2 t-1) \cdot h+(2-2 t) \cdot e ; h g], \quad t \geq \frac{1}{2} ;
\end{aligned}
$$

hence we obtain the following formulae:

$$
\begin{aligned}
H(0, g, h) & =[1 \cdot e+0 \cdot e, h g]=j_{0}(h g)=j_{0} \circ \mu \circ T(g, h), \\
H(1, g, h) & =[1 \cdot h+0 \cdot e, h g]=[1 \cdot h+0 \cdot h, g h] \\
& =\left[1 \cdot e+0 \cdot e, h^{-1}(h g) h\right]=[1 \cdot e+0 \cdot e, g h] \\
& =j_{0} \circ \mu(g, h) .
\end{aligned}
$$

This implies the desired homotopy relation.

\section{The Proof of Theorem 2.1}

The existence of a homotopy equivalence $\phi: B G \times G \rightarrow B \Lambda G$ implies that $G$ $\subseteq_{j} B \Lambda G$ is a retract up to homotopy. If so, there is a left homotopy inverse $r$ : $B \Lambda G \rightarrow G$ of $j$, and hence, $\mu \circ T \sim r \circ j \circ \mu \circ T \sim r \circ j \circ \mu \sim \mu$. By Hubbuck's Torus Theorem, this implies that $G$ has the homotopy type of a torus of some dimension $\geq 0$, since $G$ has the homotopy type of a finite CW complex. This completes the proof of the theorem.

\section{The Proof of Theorem 2.2}

The condition iv) clearly implies i). So, we firstly show the condition i) implies ii). The condition i) implies that $j_{*}: H_{*}\left(G ; \mathbb{F}_{p}\right) \rightarrow H_{*}\left(B \Lambda G ; \mathbb{F}_{p}\right)$ is injective. On the other hand, the homotopy relation given in Lemma 3.1 implies that $j_{*} \circ \mu_{*} \circ T_{*}$ $=j_{*} \circ \mu_{*}$. Hence the condition i) implies that $\mu_{*} \circ T_{*}=\mu_{*}$; in other words, the multiplication of the Pontryagin ring is commutative. Thus i) implies ii).

Secondly, we show the condition ii) implies iii). The condition ii) implies that the cohomology Hopf algebra $H^{*}\left(G ; \mathbb{F}_{p}\right)$ is bicommutative, and hence, is primitively generated, by Kane [4]. Thus ii) implies iii).

Thirdly, we show the condition iii) implies iv). The condition iii) implies that $H^{*}\left(G ; \mathbb{F}_{p}\right)$ is a biprimitive exterior algebra when $p$ odd, by Zabrodsky [9]. Then it follows that $H^{*}\left(G ; \mathbb{F}_{p}\right)$ is generated by odd-dimensional transgressive generators which determine completely the cohomology Serre spectral sequence for the universal principal $G$-bundle $\pi$. Hence we have that $H^{*}\left(B G ; \mathbb{F}_{p}\right)$ is a polynomial algebra concentrated in even dimensions:

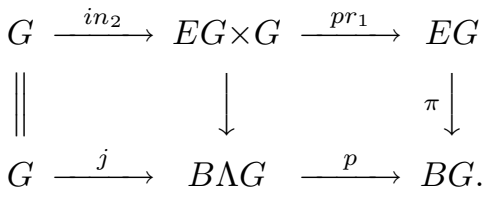

Now we consider the cohomology Serre spectral sequence for $p$. Let $x$ be a generator of the lowest dimension which is not a permanent cycle in the spectral sequence, say $d_{r} x=\sum_{a} u_{a} \otimes x_{a} \neq 0$. Then, since $H^{*}\left(G ; \mathbb{F}_{p}\right)$ is primitively generated, $x$ is an odd-dimensional primitive generator, and hence $d_{r} x$ has even total dimension. On the other hand, $p: B \Lambda G \rightarrow B G$ is a fibrewise Hopf space (see [3]), and the differential $d_{r}$ is a coalgebra homomorphism over the algebra $H^{*}\left(B G ; \mathbb{F}_{p}\right)$. This 
implies that $x_{a}$ 's are all primitive, and hence, are all odd generators, and $u_{a}$ 's are even-dimensional. This contradicts the fact that $d_{r} x$ has even total dimension. Thus all the generators are permanent cycles and hence the condition iii) implies iv). This completes the proof of Theorem 2.2.

\section{The PROOF of TheOREM 2.3}

It is sufficient to show that the conditions v), vi) and vii) are equivalent.

Firstly we show the condition vii) implies v). The condition vii) clearly implies the condition iv), and hence, ii) by Theorem 2.2. Then by Theorem D of [9] and Theorem 1.1 of [4], it follows that $H_{*}(G ; \mathbb{Z})$ has no $p$-torsion. Thus vii) implies v).

Secondly we show the condition v) implies vi). The condition v) clearly implies ii). By the condition ii), we have $a d^{*}=p r_{2}{ }^{*}: H^{*}\left(G ; \mathbb{F}_{p}\right) \rightarrow H^{*}\left(G \times G ; \mathbb{F}_{p}\right) \cong$ $H^{*}\left(G ; \mathbb{F}_{p}\right) \otimes H^{*}\left(G ; \mathbb{F}_{p}\right)$. On the other hand, by v), the cohomology suspension $\sigma^{*}$ : $H^{*}\left(G ; \mathbb{F}_{p}\right)$ induces an isomorphism $Q H^{*}\left(G ; \mathbb{F}_{p}\right) \cong P H^{*}\left(\Omega G ; \mathbb{F}_{p}\right)$ with the following relation:

$$
A d^{*} \sigma^{*}=\left(1 \otimes \sigma^{*}\right) a d^{*}=\left(1 \otimes \sigma^{*}\right) p r_{2}{ }^{*}=p r_{2}{ }^{*} \sigma^{*} .
$$

Hence, $A d^{*}=p r_{2}{ }^{*}$ on the module of primitive elements $P H^{*}\left(\Omega G ; \mathbb{F}_{p}\right)$. Then for a generator $u \in P H_{*}\left(\Omega G ; \mathbb{F}_{p}\right)$ and an element $x \in H_{*}\left(G ; \mathbb{F}_{p}\right)$, we have the equation

$$
A d_{*}(x \otimes u)=p r_{2 *}(x \otimes u)=0
$$

modulo the module of decomposables $D H^{*}\left(\Omega G ; \mathbb{F}_{p}\right)$. On the other hand, since

$$
\begin{aligned}
& A d(1 \times A d)\left(g_{1}, g_{2}, h\right)=A d\left(g_{1}, g_{2} h g_{2}^{-1}\right) \\
& \quad=g_{1} g_{2} h g_{2}^{-1} g_{1}^{-1}=A d\left(g_{1} g_{2}, h\right)=A d(\mu \times 1)\left(g_{1}, g_{2}, h\right),
\end{aligned}
$$

one has the relation $A d(1 \times A d)=A d(\mu \times 1)$, where we denote by $\mu$ the multiplication of the group $G$. Using this, by the induction on the dimension of an element in $H_{*}\left(\Omega G ; \mathbb{F}_{p}\right)$, we have the desired formula: $A d_{*}(x \otimes u)=0$ for $x \in \tilde{H}_{*}\left(G ; \mathbb{F}_{p}\right)$. This implies that $A d^{*}(u)=1 \otimes u$. Thus the condition v) implies vi).

Finally, we show the condition vi) implies vii). Assuming that $H_{*}\left(G ; \mathbb{F}_{p}\right)$ is not commutative, under the condition vi), we shall be led to a contradiction. Let $[a, b]$ be a nonzero commutator in the lowest dimension, say $m$. Since $H_{*}\left(G ; \mathbb{F}_{p}\right)$ is associative, it follows that $a$ and $b$ are generators and $[a, b]$ is primitive. Then by Theorem 5.4 .1 (c) of [6], the $m$ must be odd, and hence, we may assume that $a$ is an even-dimensional generator and $b$ is an odd-dimensional generator. Let us dualize the situation: Let $u$ and $v$ be the dual primitive elements to $a$ and $b$, and choose $x$ to be a generator such that $\langle x,[a, b]\rangle \neq 0$. Since $H^{*}\left(G ; \mathbb{F}_{p}\right)$ is associative and commutative, by Proposition 4.21 of [7], $v$ is an odd primitive generator. Then the diagonal image $\tilde{\mu}^{*}(x)$ satisfies $\left\langle\tilde{\mu}^{*}(x), a \otimes b\right\rangle \neq 0$. Here, by a series of results on the cohomology of a Hopf space such as those given in [8], [1] or [2], it follows that $\sigma^{*}: Q H^{\text {odd }}\left(G ; \mathbb{F}_{p}\right) \rightarrow P H^{\text {even }}\left(\Omega G ; \mathbb{F}_{p}\right)$ is injective. Hence we have that $\sigma^{*}(x) \neq 0$, $\sigma^{*}(v) \neq 0$ and $\sigma^{*}(u)=0$. Also we have the relation

$$
\begin{aligned}
A d^{*}\left(\sigma^{*}(x)\right) & =A d^{*} \sigma^{*}(x)=\left(1 \otimes \sigma^{*}\right) a d^{*}(x)=\left(1 \otimes \sigma^{*}\right)\left(p r_{2}{ }^{*} x+\tilde{\mu^{*}}(x)\right) \\
& =\left(1 \otimes \sigma^{*}\right) p r_{2}{ }^{*}(x)+\left(1 \otimes \sigma^{*}\right)\left(\tilde{\mu_{*}}(x)\right) \\
& =p r_{2}{ }^{*} \sigma^{*}(x) \pm u \otimes \sigma^{*}(v) \neq p r_{2}{ }^{*} \sigma^{*}(x) .
\end{aligned}
$$

This implies that $A d^{*} \neq p r_{2}{ }^{*}$. Thus the condition vi) implies the condition ii), and hence iii) and iv). Therefore, it implies the existence of an $H^{*}\left(B G ; \mathbb{F}_{p}\right)$-module 
isomorphism $H^{*}\left(B \Lambda G ; \mathbb{F}_{p}\right) \cong H^{*}\left(B G ; \mathbb{F}_{p}\right) \otimes H^{*}\left(G ; \mathbb{F}_{p}\right)$, where $H^{*}\left(G ; \mathbb{F}_{p}\right)$ is generated by odd primitive elements. Then the square of the odd-dimensional generators of $H^{*}\left(G ; \mathbb{F}_{p}\right)$ in $H^{*}\left(B \Lambda G ; \mathbb{F}_{p}\right)$ must be trivial by the standard argument of graded commutative algebras, and hence the isomorphism preserves the algebra structures. Thus the condition vi) implies vii).

\section{REFERENCES}

1. W. Browder, On differential Hopf algebras, Trans. Amer. Math. Soc. 107 (1963), 153-176. MR 26:3061

2. A. Clark, Homotopy commutativity and the Moore spectral sequence, Pacific. J. Math. 15 (1965), 65-74. MR 31:1679

3. A.L. Cook and M.C. Crabb, Fibrewise Hopf structures on sphere-bundles, J. London Math. Soc. 48 (1993), 365-384. MR 94d:55021

4. R. Kane, Primitivity and finite H-spaces, Q. J. Math. Oxford 3 (1975), 185-197. MR 52:9220

5. K. Kono and K. Kozima, The adjoint action of a Lie group on the space of loops, J. Math. Soc. Japan 45 (1993), 495-509. MR 94h:57053

6. J.P. Lin, Torsion in H-spaces II, Ann. Math. 107 (1978), 41-88. MR 58:7619

7. J. Milnor and J.C. Moore, On the structure of Hopf Algebras, Ann. Math. 81 (1965), 211-264. MR 30:4259

8. J.C. Moore, La Suspension, Seminar H. Cartan 12 (1959/60), expose 6.

9. A. Zabrodsky, Implications in the cohomology of H-space, Illinois J. Math. 16 (1971), 363-375. MR 41:6217

Graduate School of Mathematics Ropponmatsu, Kyushu University, Ropponmatsu 42-1, FuKUOKA 810, JAPAN

E-mail address: iwase@math.kyushu-u.ac.jp 